\title{
Racial and ethnic disparities in diabetes risk after gestational diabetes mellitus
}

\author{
A. H. Xiang • B. H. Li • M. H. Black • D. A. Sacks • \\ T. A. Buchanan • S. J. Jacobsen • J. M. Lawrence
}

Received: 15 July 2011 / Accepted: 9 September 2011 /Published online: 21 October 2011

(C) Springer-Verlag 2011

\begin{abstract}
Aims/hypothesis To investigate racial/ethnic disparities in diabetes risk after gestational diabetes mellitus (GDM).

Methods This is a retrospective cohort study of women enrolled in the Kaiser Permanente Southern California health plan from 1995 to 2009. GDM status was identified on the basis of plasma glucose levels during pregnancy. The incidence of diabetes after the first delivery complicated by GDM before 31 December $2009(n=12,998)$ was compared with the experience for women without $\operatorname{GDM}(n=64,668)$ matched on maternal age at delivery, race/ethnicity and year of delivery (1:5 ratio). Matched Cox regression was used to compare the RRs of diabetes associated with GDM within and across racial/ethnic groups.

Results Compared with the women without GDM, the HRs $(95 \% \mathrm{CI})$ of diabetes for women after GDM were 6.5 (5.2, 8.0) in non-Hispanic white, $7.7(6.8,8.7)$ in Hispanic, 9.9 $(7.5,13.1)$ in black and $6.3(5.0,7.9)$ in Asian/Pacific Islanders after adjustment for parity, maternal education, comorbidity and number of outpatient visits before the index pregnancy. The HR of diabetes for black women was significantly higher than that for non-Hispanic white women $(p=0.032)$. Further adjustment for prepregnancy BMI reduced the diabetes risk association with GDM for
\end{abstract}

A. H. Xiang $(\bowtie) \cdot$ B. H. Li • M. H. Black • D. A. Sacks •

S. J. Jacobsen - J. M. Lawrence

Department of Research \& Evaluation,

Kaiser Permanente Southern California,

100 S. Los Robles, 5th Floor,

Pasadena, CA 91101, USA

e-mail: anny.h.xiang@kp.org

T. A. Buchanan

Department of Medicine Keck School of Medicine,

University of Southern California,

Los Angeles, CA, USA each racial/ethnic group, but did not explain the risk differences across groups.

Conclusions/interpretations Racial/ethnic disparities exist in risk of diabetes after GDM. Black women with GDM had the highest risk of developing diabetes. This highlights the importance of developing an effective diabetes screening and prevention programme in women with GDM, particularly black women with GDM.

Keywords Diabetes risk - Gestational diabetes mellitus . Longitudinal follow-up · Racial/ethnic difference

$\begin{array}{ll}\text { Abbreviations } \\ \text { API } & \text { Asian/Pacific Islanders } \\ \text { GCT } & \text { Glucose challenge test } \\ \text { GDM } & \text { Gestational diabetes mellitus } \\ \text { KPSC } & \text { Kaiser Permanente Southern California } \\ \text { LMP } & \text { Last menstrual period } \\ \text { NHW } & \text { Non-Hispanic white }\end{array}$

\section{Introduction}

Gestational diabetes mellitus (GDM) is a state of glucose intolerance first detected during pregnancy $[1,2]$. The prevalence of GDM varies by race/ethnicity [3-6], with the highest prevalence reported in Asian/Pacific Islanders (API) and relatively low prevalence in black people $[3,4,6]$. The prevalence of GDM has increased across all race/ethnic groups $[4,7]$. GDM is associated with short-term and longterm adverse outcomes for both mothers and their offspring. In particular, women with GDM have a $20-60 \%$ risk of developing diabetes in the 5-10 years after the index pregnancy $[8,9]$. Current knowledge about the incidence of 
diabetes after GDM is based mostly on small studies and/or racially/ethnically homogeneous populations. A recent systematic review and meta-analysis concluded that women with GDM had a sevenfold increased risk of developing type 2 diabetes compared with women without GDM [10]. Moreover, the authors concluded that such risk appeared to be similar across all ethnic groups despite large variations in the RR among studies.

To address whether differences in GDM prevalence across race/ethnicity translate into similar disparities in overt diabetes conversion after GDM across race/ethnicity, we conducted a large retrospective matched cohort study in a racially/ethnically diverse population. The purpose of this study was to investigate racial/ethnic disparities in diabetes risk after GDM.

\section{Methods}

Population and data sources The study cohort comprised women who had a singleton delivery at $\geq 20$ weeks gestation in Kaiser Permanente Southern California (KPSC) hospitals between 1 January 1995 and 31 December 2008. KPSC is an integrated healthcare system that provides comprehensive prepaid health services for its over 3 million members in 2011. Members are demographically/socioeconomically diverse. Study data were extracted from multiple clinical and administrative data systems and from infant birth certificates. Pregnancy and delivery-related information (maternal age, education and race/ethnicity; last menstrual period [LMP], parity and date of delivery) were obtained from the infant birth certificates. Maternal race/ ethnicity was categorised as Hispanic (regardless of race), non-Hispanic white (NHW), black, API and 'other', which included women with other, multiple or unknown race. Maternal comorbidity was defined as one or more diagnoses of heart, lung, kidney or liver disease or cancer based on inpatient and outpatient encounter codes before index delivery. Prepregnancy medical utilisation was defined as the number of outpatient visits in the year before the LMP of the index pregnancy. Prepregnancy height and weight, when available, were extracted from the electronic health record. This study was approved by the KPSC Institutional Review Board.

Identification of women with GDM Women who had known type 1 diabetes (ICD-9 [www.icd9data.com/2007/ Volume1/240-279/250-259/250/default.htm] of 250.X1 and 250.X3) or existing diabetes before the study entry were excluded. Women were classified as having GDM on the basis of their plasma glucose levels during pregnancy according to the following hierarchy: (1) at least two levels meeting or exceeding the Carpenter and Coustan threshold values [11] (fasting $\geq 5.3,1 \mathrm{~h} \geq 10.0,2 \mathrm{~h} \geq 8.6$ and $3 \mathrm{~h} \geq$ $7.8 \mathrm{mmol} / \mathrm{l}$ ) during the $100 \mathrm{~g}, 3 \mathrm{~h}$ OGTT; or (2) at least two levels during the $75 \mathrm{~g}, 2 \mathrm{~h}$ OGTT greater than or equal to the same threshold values [11]; or (3) a single level of $1 \mathrm{~h}$, $50 \mathrm{~g}$ glucose challenge test $(\mathrm{GCT}) \geq 11.1 \mathrm{mmol} / \mathrm{l}$.

Identification of incident diabetes Non-pregnant women were classified as having diabetes if they met at least one of the following criteria after the index pregnancy: (1) plasma glucose levels meeting the American Diabetes Association diabetes diagnosis criteria (fasting $\geq 7.0 \mathrm{mmol} / 1$ or $2 \mathrm{~h} \geq$ $11.1 \mathrm{mmol} / 1$ from $75 \mathrm{~g}$ OGTT, or a random level $\geq 11.1 \mathrm{mmol} /$ 1 , where fasting and $2 \mathrm{~h}$ were confirmed on a separate day with a repeat test within 6 months) [11]; or (2) $\mathrm{HbA}_{1 \mathrm{c}}$ level $\geq 7.0 \%$ $(53 \mathrm{mmol} / \mathrm{mol})$ or (3) a prescription of insulin or other oral glucose-lowering drugs; or (4) an ICD-9 code of 250.xx from one or more inpatient encounters, or two or more outpatient encounters within 2 years. Women who took metformin who were also diagnosed as having polycystic ovary syndrome (ICD-9 256.xx) and had no other indicator of diabetes were not considered to have diabetes.

Subject selection and follow-up Women were followed passively through their electronic health records for a diagnosis of diabetes. Follow-up started on the index delivery date for women with and without GDM. To minimise bias due to disenrollment and to assess relatively long-term diabetes risk, all women were required to have at least 1 year of continuous health plan membership (allowing gaps for up to 90 days) before the LMP before the index pregnancy and at least 1 year of continuous membership (allowing gaps for up to 90 days) after their index delivery. Follow-up ended on the first date that any one of the following criteria was met: (1) a diagnosis of diabetes; (2) death from any cause; (3) last date where a gap of greater than 4 months of inactive health plan membership occurred; or (4) 31 December 2009, the end of the study period. A total of 139,344 women met these continuous membership criteria, from which a matched-pair cohort was derived. Each woman with GDM was matched to five women without GDM by random sampling of all eligible women without GDM matching on race/ethnicity, age ( \pm 3 years) and calendar year of study entry. Women with GDM who could not be matched to any suitable women without GDM were not included. Overall, 91\% of women with GDM were included in the matched cohort.

Statistical data analysis Parity at the time of the index pregnancy was categorised into $0,1, \geq 2$ or missing. Education was classified into $\leq$ high school graduate, some college, $\geq$ college graduate, or missing. History of any comorbid condition was classified as present or absent (yes/ no). Crude incidence of diabetes was calculated by number of women who developed diabetes divided by the total 
number of person-years of follow-up. Crude rate ratio and $95 \%$ CI comparing women with and without GDM by race/ ethnicity were computed and tested for statistical significance by Poisson regression. Cumulative incidence rates of diabetes by race/ethnicity and maternal GDM status were estimated by the Kaplan-Meier method. Matched Cox regression models were used to estimate the adjusted (for parity, education, presence of comorbidity and prepregnancy medical utililsation) HRs of developing diabetes after GDM. The few women with missing parity and/or education data were included in all data analyses by including missing indicator variables as covariates in the adjusted analyses. The Cox regression analyses were first performed within each racial/ethnic group to estimate the covariate-adjusted racial/ethnic specific HR of developing diabetes associated with GDM. To assess whether HRs differed across racial/ethnic groups, Cox regression analyses were then performed by combining data from all groups with NHW women as the reference group and testing for significant first-order interaction between GDM status and other racial/ethnic group indicator.

We performed additional analyses to assess whether obesity could explain the racial/ethnic disparities in the risk of developing diabetes after GDM. Index prepregnancy BMI data were available for only $13,118(16.7 \%)$ of the women in the matched cohort because of the roll-out of the electronic health record across the region starting in 2006. We imputed missing BMI by multiple imputations where known index pregnancy characteristics were incorporated with the Markov Chain Monte Carlo approach. This is a simulation-based approach for estimating the missing data from the predicted distributions on the basis of available observed data. The approach produces valid estimates when data are missing at random. Twenty imputed datasets were created. Matched Cox regression was performed for each of the imputed datasets, with prepregnancy BMI included in the models in addition to other index pregnancy characteristics. The results were combined using Rubin's rule to incorporate the variations across the 20 imputed datasets [12].

SAS Enterprise Guide 4.3 (SAS Institute Inc, Cary, NC, USA) was used for data analysis. PROC MI and PROC MIANALYZE were used for multiple imputations. All statistical tests were two-sided, and statistical significance was defined as $p \leq 0.05$.

\section{Results}

Characteristics of women with and without GDM A total of 139,334 women met the singleton pregnancy and KPSC membership duration criteria for consideration for the study; 14,299 (10.3\%) were identified as having GDM
(81.6\% by $100 \mathrm{~g} 3 \mathrm{~h}$ OGTT, $9.0 \%$ by $75 \mathrm{~g} 2 \mathrm{~h}$ OGTT, and $9.4 \%$ by $50 \mathrm{~g} 1 \mathrm{~h} \mathrm{GCT)}$. GDM was most prevalent in API $(17.1 \%)$ and Hispanic (11.4\%) women, while GDM prevalence was comparable among NHW (7.4\%), black $(6.9 \%)$ and other $(7.7 \%)$ women. Across all racial/ethnic groups, women with GDM were older and had higher parity than women without GDM (data not shown).

Of the 14,299 eligible women with GDM, 12,998 (91\%) were successfully matched by age, race/ethnicity and calendar year of entry to a total of 64,668 women without GDM (i.e. $52 \%$ of the eligible controls). The remaining $9 \%$ of women with GDM could not be matched to appropriate controls. In the matched cohort (Table 1), there were small but statistically significant differences between GDM exposed and unexposed in parity, education, presence of comorbidities, and prepregnancy visits. Age differed by only 0.1 year. Race/ethnicity was well matched between the two groups.

Incident diabetes In the matched cohort, a total of 2,657 women developed diabetes during 439,584 person-years of follow-up, which included $456 \mathrm{NHW}, 1,464$ Hispanic, 309 black, 403 API and 25 women of other race/ethnicity. The

Table 1 Baseline characteristics of women with and without GDM in the matched cohort

\begin{tabular}{llll}
\hline Characteristic & $\begin{array}{l}\text { GDM } \\
(n=12,998)\end{array}$ & $\begin{array}{l}\text { Non-GDM } \\
(n=64,668)\end{array}$ & $p$ value \\
\hline $\begin{array}{llll}\text { Age (years), mean } \pm \text { SD } \\
\text { Parity, } n(\%)\end{array}$ & $32.4 \pm 5.2$ & $32.3 \pm 5.1$ & 0.002 \\
0 & $4,544(35.0)$ & $29,422(45.5)$ & $<0.0001$ \\
1 & $3,899(30.0)$ & $16,807(26.0)$ & \\
$\geq 2$ & $4,432(34.1)$ & $17,847(27.6)$ & \\
Missing & $123(1.0)$ & $592(0.9)$ & \\
Race/ethnicity, $n(\%)$ & & & 0.99 \\
NHW & $2,902(22.3)$ & $14,498(22.4)$ & \\
Hispanic & $6,673(51.3)$ & $33,245(51.4)$ & \\
Black & $1,184(9.1)$ & $5,890(9.1)$ & \\
API & $2,096(16.1)$ & $10,345(16.0)$ & \\
Other & $143(1.1)$ & $690(1.1)$ & \\
Education, $n$ (\%) & & & $<0.0001$ \\
$\leq$ High school & $5,301(40.8)$ & $23,355(36.1)$ & \\
Some college & $3,487(26.8)$ & $16,469(25.5)$ & \\
$\geq$ College graduate & $3,619(27.8)$ & $21,691(33.5)$ & \\
Missing & $591(4.6)$ & $3,153(4.9)$ & \\
Presence of comorbidity, $n(\%)$ & $1,091(8.4)$ & $4,689(7.3)$ & $<0.0001$ \\
Yes & $5.6 \pm 6.0$ & $5.1 \pm 5.8$ & $<0.0001$ \\
Number of prepregnancy & & & \\
outpatient visits, mean $\pm S D$ & & & \\
\hline
\end{tabular}

Percentages are column percentages 
Table 2 Crude diabetes incidence rates and rate ratios associated with GDM in the matched cohort

\begin{tabular}{|c|c|c|c|c|c|c|c|c|c|c|}
\hline \multirow{2}{*}{$\begin{array}{l}\text { Race/ } \\
\text { ethnicity }\end{array}$} & \multicolumn{4}{|c|}{$\operatorname{GDM}(n=12,998)$} & \multicolumn{4}{|c|}{ Non-GDM $(n=64,668)$} & \multirow{2}{*}{$\begin{array}{l}\text { Rate ratio } \\
(95 \% \mathrm{CI})\end{array}$} & \multirow[t]{2}{*}{$p$ value $^{\mathrm{a}}$} \\
\hline & $\begin{array}{l}\text { Women } \\
(n)\end{array}$ & $\begin{array}{l}\text { Diabetes } \\
\text { cases }(n)\end{array}$ & $\begin{array}{l}\text { Total person- } \\
\text { years }\end{array}$ & $\begin{array}{l}\text { Incidence } / 1,000 \\
\text { person-years } \\
(95 \% \mathrm{CI})\end{array}$ & $\begin{array}{l}\text { Women } \\
(n)\end{array}$ & $\begin{array}{l}\text { Diabetes } \\
\text { cases }(n)\end{array}$ & $\begin{array}{l}\text { Total person- } \\
\text { years }\end{array}$ & $\begin{array}{l}\text { Incidence } / 1,000 \\
\text { person-years } \\
(95 \% \mathrm{CI})\end{array}$ & & \\
\hline NHW & 2,902 & 261 & 16,527 & $15.8(14.0,7.8)$ & 14,498 & 195 & 86,110 & $2.3(2.0,2.6)$ & $7.0(5.8,8.4)$ & $<0.0001$ \\
\hline Hispanic & 6,673 & 849 & 34,525 & $24.6(23.0,26.3)$ & 33,245 & 615 & 183,872 & $3.3(3.1,3.6)$ & $7.4(6.6,8.2)$ & $<0.0001$ \\
\hline Black & 1,184 & 195 & 6,725 & $29.0(25.2,33.4)$ & 5,890 & 114 & 36,061 & $3.2(2.6,3.8)$ & $9.2(7.2,11.7)$ & $<0.0001$ \\
\hline API & 2,096 & 219 & 11,565 & $18.9(16.6,21.6)$ & 10,345 & 184 & 60,053 & $3.1(2.7,3.5)$ & $6.2(5.1,7.6)$ & $<0.0001$ \\
\hline Other & 143 & 15 & 702 & $21.4(12.9,35.4)$ & 690 & 10 & 3,444 & $2.9(1.6,5.4)$ & $7.4(3.1,18.3)$ & $<0.0001$ \\
\hline Overall & 12,998 & 1,539 & 70,044 & $22.0(20.9,23.1)$ & 64,668 & 1,118 & 369,540 & $3.0(2.8,3.2)$ & $7.3(6.7,7.9)$ & $<0.0001$ \\
\hline
\end{tabular}

${ }^{\mathrm{a}} p$ value testing the rate ratio from 1

corresponding median years of follow-up were 4.5, 5.2, 4.4, 4.8 and 3.9, respectively. The crude incidences of diabetes, stratified by GDM status and race/ethnicity, along with the corresponding rate ratios comparing women with and without GDM, are presented in Table 2. The diabetes incidence rates varied by race/ethnicity and GDM status. Black women with GDM had the highest rate of developing diabetes (29 cases per 1,000 person-years of follow-up). This rate was 9.2-fold higher $(95 \%$ CI $7.2,11.7, p<0.001)$ than in the similar age black women without GDM (3.2 cases per 1,000 person-years of follow-up), representing the highest RR of diabetes associated with GDM among all racial/ethnic groups.

Figure 1a depicts the Kaplan-Meier plot of cumulative risk of diabetes in women stratified by GDM status and race/ethnicity. HRs from matched Cox regression analysis with adjustment for parity, education, presence of comorbidity and number of prepregnancy outpatient visits are shown in Fig. 1b. After adjustment for these covariates, the HRs $(95 \% \mathrm{CI})$ of developing diabetes after GDM were 6.5 $(5.2,8.0)$ in NHW, $7.7(6.8,8.7)$ in Hispanic, $9.9(7.5,13.1)$ in black, $6.3(5.0,7.9)$ in API, and $10.5(3.3,33.3)$ in other women. The risk in black women was $52 \%$ higher than that in NHW ( $p=0.032$ from the interaction term testing for difference in HRs). The RR in Hispanic, API and other women was not significantly different from the RR for NHW women ( $p=0.21,0.76$ and 0.54 , respectively).

Impact of obesity on the disparities in risk of diabetes associated with GDM Table 3 presents the number of women with measured prepregnancy BMI data and differences in mean observed BMI between women with and without GDM by racial/ethnic group. The average prepregnancy BMI was lowest in API women and highest in black women. In addition, BMI in women with GDM was 2-4 units higher than in women without GDM across all racial/ ethnic groups $(p \leq 0.0004)$. After imputation of missing BMI data, the matched Cox regression models further adjusted for maternal BMI demonstrated that the association between GDM and diabetes was attenuated in all racial/ethnic groups (Fig. 1b). However, the difference in BMI did not account for the entire excess risk of diabetes
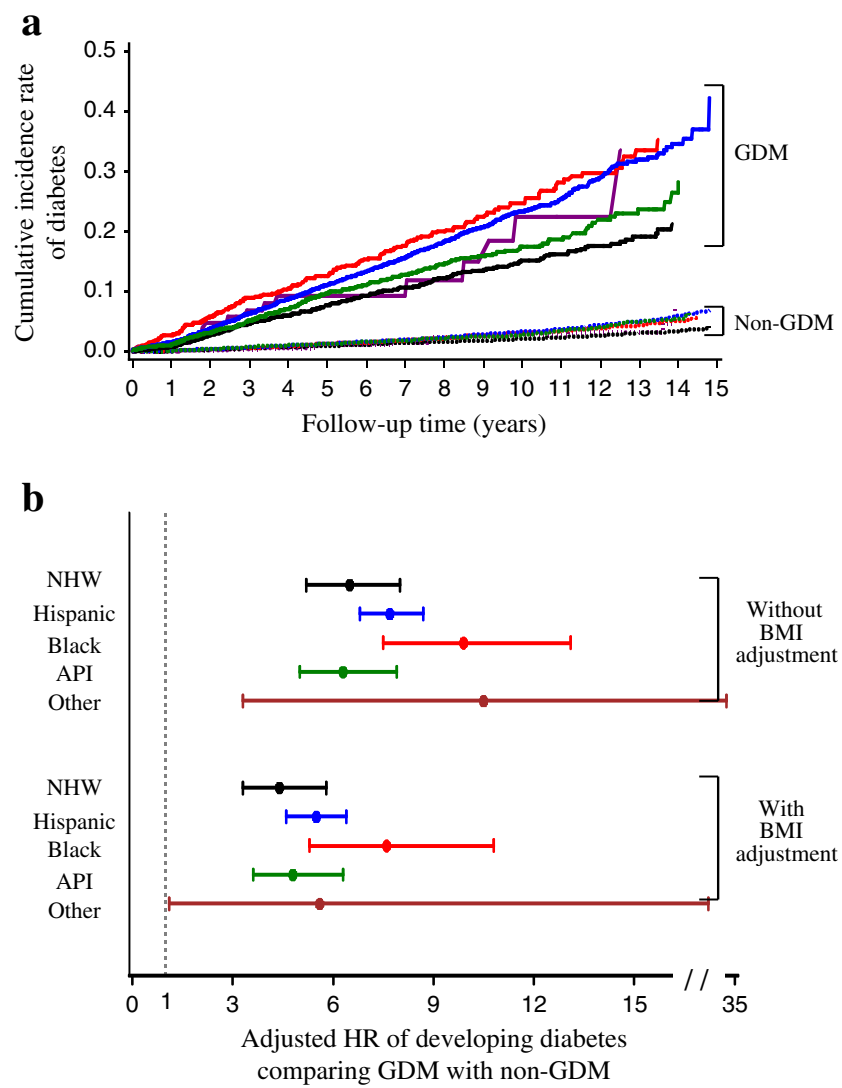

Fig. 1 Kaplan-Meier plot of diabetes cumulative incidence rates stratified by GDM status and race/ethnicity (a) and adjusted HR of developing diabetes comparing GDM with non-GDM by race/ ethnicity (b). The horizontal line in b is the $95 \%$ CI. The $p$ value tests whether the HR in other racial/ethnic groups is significantly different from that in NHW. All $p$ values for comparison with NHW are not significant $(p>0.05)$ except for Black without $(p=0.032)$ and with $(p=0.028)$ BMI adjustment. Black, NHW; blue, Hispanic; red, black; green, API; purple, other 
Table 3 Comparison of available baseline prepregnancy BMI for the matched cohort

${ }^{a}$ Testing the difference in mean BMI between GDM and nonGDM within each racial/ethnic group

\begin{tabular}{|c|c|c|c|c|c|c|}
\hline \multirow{2}{*}{$\begin{array}{l}\text { Women with } \\
\text { BMI data }\end{array}$} & \multicolumn{2}{|l|}{ GDM } & \multicolumn{2}{|c|}{ Non-GDM } & \multirow{2}{*}{$\begin{array}{l}\text { Difference in } \\
\text { mean BMI }\end{array}$} & \multirow[t]{2}{*}{$p$ value } \\
\hline & $n$ & Mean \pm SD & $n$ & Mean \pm SD & & \\
\hline NHW & 414 & $29.2 \pm 7.2$ & 2,127 & $25.8 \pm 5.9$ & 3.4 & $<0.0001$ \\
\hline Hispanic & 1,188 & $30.7 \pm 6.4$ & 5,901 & $27.4 \pm 5.9$ & 3.3 & $<0.0001$ \\
\hline Black & 166 & $32.1 \pm 8.5$ & 838 & $29.0 \pm 7.1$ & 3.1 & $<0.0001$ \\
\hline API & 376 & $25.2 \pm 4.9$ & 1,897 & $23.2 \pm 4.1$ & 2.0 & $<0.0001$ \\
\hline Other & 40 & $30.5 \pm 7.4$ & 171 & $26.5 \pm 6.0$ & 4.0 & 0.0004 \\
\hline
\end{tabular}

associated with GDM, and it did not account for the difference in $\mathrm{RR}$ across racial/ethnic groups. GDM remained a significant risk factor for diabetes in every racial/ethnic group with HRs ranging from 4.4 to 7.6 . The $\mathrm{RR}$ in black women remained significantly higher than that in NHW women (7.6 vs 4.4, $p=0.028$ ). The corresponding $p$ values comparison of Hispanic, API and other women with NHW women were $0.20,0.70$ and 0.88 , respectively.

\section{Discussion}

In this systematic assessment of racial/ethnic disparities in the development of diabetes after GDM in a large diverse cohort of insured women, diabetes risk associated with GDM was shown to vary by maternal race/ethnicity. Despite the fact that the prevalence of GDM in black women was low relative to women from other racial/ethnic minority groups, as demonstrated in other studies [4, 6, 7], black women who developed GDM had the highest risk of developing diabetes after GDM, independent of age, parity, education, comorbidity status, prepregnancy medical utilisation and BMI. Similarly, although the GDM prevalence was the highest among API women, the risk of developing diabetes after GDM was comparable to that in NHW women.

The overall RR of developing diabetes associated with GDM in our study was 7.3, which is consistent with the meta-analysis result of Bellamy et al. [10], which included 20 studies published from 1960 through January 2009 that included 675,455 women with and without GDM and 10,859 diabetes events. The pooled estimate of RR of diabetes associated with GDM was 7.4 with variation across studies. However, as the authors pointed out, they had little capability to evaluate the variations across race/ ethnicity because they did not have individual-level data. Our study is based on individual-level data collected uniformly across women in a large multi-ethnic cohort. We demonstrated that there is significant variation in the RR and absolute risk of developing diabetes after GDM across racial/ethnic groups, where risk is the highest in black women. Another large sample population-based study assessing RR of developing diabetes associated with GDM was based on the Ontario Diabetes Database by Feig et al. [13]. The authors reported an adjusted HR of 37.3 (95\% CI 35.0, 40.9) associated with GDM. However, much of the high risk was due to the greatest risk in the first year post partum, which may also include pre-existing undiagnosed diabetes from women with GDM (Fig. 2 of Feig et al.). After the first year, the unadjusted RR is $\sim 7.2$ (estimated from their Fig. 2), which is similar to our estimate. Race/ ethnicity distribution was not reported in that study.

We acknowledge several potential limitations of our study. First, pre-index pregnancy BMI data were available for only $\sim 17 \%$ of the cohort because of the timing of electronic health record implementation across medical centres within KPSC. It is likely that the data were missing at random, and our use of multiple imputations should provide valid estimates. In addition, follow-up time was truncated by health plan disenrollment, at which point subjects were censored in the survival analysis. However, it is reasonable to assume that disenrollment is unrelated to diabetes risk in this relatively young cohort of women of reproductive age. Thus, censoring was non-informative and the use of survival analysis was appropriate. Finally, diabetes incidence rates may be underestimated because of sub-clinical diabetes which would not be found in the medical record. The diabetes incidence rate was $2.5 \%$ /year in Hispanic women with GDM in this cohort (Table 2), which was much lower than the $7.2 \%$ /year or the $11 \%$ /year observed previously in smaller cohort studies of Hispanic women with GDM [9, 14]. The higher incidence rates in these other cohorts may be due to frequently scheduled OGTT testing and the inclusion of higher-risk women. Since uniform criteria were applied for all racial/ethnic groups to identify GDM and diabetes status in this study, underestimating diabetes cases should have minimal impact on our conclusions of racial/ethnic disparities in the risk of developing diabetes after GDM.

The strengths of this study include our large, racially/ ethnically diverse cohort that is well characterised in terms of GDM status, and our ability to identify subsequent diabetes from a clinical patient care system that provided a rich source of laboratory and pharmacy data. Our membership retention 
allowed us to obtain a relatively long-term follow-up, and our matched study design afforded us greater control for the important confounding effects of age, race/ethnicity and secular trend. Owing to the availability of large samples of women without GDM, we were able to use a matched-pair cohort design to include the maximum number of women with GDM (91\% of all eligible women with GDM), and simultaneously minimise confounding bias by matching without sacrificing study power. We conducted analyses including all 139,344 eligible women and adjusted for age, race/ethnicity and calendar year of entry in addition to parity, education, presence of comorbidity and number of prepregnancy outpatient visits by using covariate adjustment. After recognition that residual confounding could be present, the adjusted HRs were found to be 7.2 $(6.1,8.4)$ in NHW, $7.4(6.7$, 8.2) in Hispanic, $9.0(7.5,10.9)$ in black and $6.1(5.1,7.4)$ in API women. Compared with NHW, the HR was significantly higher for black $(p=0.02)$, but not for Hispanic $(p=0.47)$ or API $(p=0.33)$ women. These results are consistent with the matched cohort, supporting our conclusions from the matched cohort analysis.

In summary, in a large, multi-ethnic cohort study within an integrated managed healthcare system, we found racial/ ethnic disparities in diabetes risk after GDM. Despite relatively low GDM prevalence among black women, their risk of developing diabetes associated with GDM was the highest among all racial/ethnic groups. Whether this difference is due to genetics, environment, lifestyle or other differences among ethnic groups will require further investigation. We conclude that race and ethnicity should be considered in counselling women about their risk of diabetes after a pregnancy complicated by GDM in clinical care. Our results highlight the importance of developing an effective diabetes screening and prevention programme in women with GDM, particularly black women with GDM.

Acknowledgements This work was supported by Kaiser Permanente Southern California Direct Community Benefit funds.

Contribution statement All authors contributed to the conception and design, or analysis and interpretation of data; drafted the article or revised it critically for important intellectual content; and have final approval of the version to be published.
Duality of interest All the authors declare that they have no duality of interest associated with this manuscript.

\section{References}

1. American Diabetes Association (2011) Standards of medical care in diabetes-2011. Diabetes Care 34(Suppl 1):S11-S61

2. Buchanan TA, Xiang A, Kjos SL, Watanabe R (2007) What is gestational diabetes? Diabetes Care 30(Suppl 2):S105-S111

3. Berkowitz GS, Lapinski RH, Wein R, Lee D (1992) Race/ ethnicity and other risk factors for gestational diabetes. Am J Epidemiol 135:965-973

4. Lawrence JM, Contreras R, Chen W, Sacks DA (2008) Trends in the prevalence of preexisting diabetes and gestational diabetes mellitus among a racially/ethnically diverse population of pregnant women, 1999-2005. Diabetes Care 31:899-904

5. Hedderson MM, Darbinian JA, Ferrara A (2010) Disparities in the risk of gestational diabetes by race-ethnicity and country of birth. Paediatr Perinat Epidemiol 24:441-448

6. Ferrara A, Kahn HS, Quesenberry CP, Riley C, Hedderson MM (2004) An increase in the incidence of gestational diabetes mellitus: Northern California, 1991-2000. Obstet Gynecol 103:526-533

7. Dabelea D, Snell-Bergeon JK, Hartsfield CL, Bischoff KJ, Hamman RF, McDuffie RS (2005) Increasing prevalence of gestational diabetes mellitus (GDM) over time and by birth cohort: Kaiser Permanente of Colorado GDM Screening Program. Diabetes Care 28:579-584

8. Kim C, Newton KM, Knopp RH (2002) Gestational diabetes and the incidence of type 2 diabetes: a systematic review. Diabetes Care 25:1862-1868

9. Kjos SL, Peters RK, Xiang A, Henry OA, Montoro M, Buchanan TA (1995) Predicting future diabetes in Latino women with gestational diabetes. Utility of early postpartum glucose tolerance testing. Diabetes 44:586-591

10. Bellamy L, Casas JP, Hingorani AD, Williams D (2009) Type 2 diabetes mellitus after gestational diabetes: a systematic review and meta-analysis. Lancet 373:1773-1779

11. Association AD (2010) Diagnosis and classification of diabetes mellitus. Diabetes Care 33(Suppl 1):S62-S69

12. Rubin DB (1987) Multiple imputation for nonresponse in surveys. Wiley, New York

13. Feig DS, Zinman B, Wang X, Hux JE (2008) Risk of development of diabetes mellitus after diagnosis of gestational diabetes. CMAJ 179:229-234

14. Xiang AH, Kjos SL, Takayanagi M, Trigo E, Buchanan TA (2010) Detailed physiological characterization of the development of type 2 diabetes in Hispanic women with prior gestational diabetes mellitus. Diabetes 59:2625-2630 